\title{
Report of a rare fistula between a Studer neobladder and external iliac artery
}

\author{
Nicholas J. Kuntz, MD; Brant A. Inman, MD
}

Division of Urology, Duke University Medical Center, Durham, NC

Cite as: Can Urol Assoc J 2013;7(9-10):e645-7. http://dx.doi.org/10.5489/cuaj.402

Published online October 9, 2013.

\section{Abstract}

A neobladder-arterial fistula is a very rare complication following cystectomy, with only 1 previously reported case. Delay in diagnosis can be rapidly fatal and requires prompt intervention. We report the case of a 63-year-old male who developed massive hematuria, and was found to have a fistula between the right external iliac artery and Studer neobladder during emergent exploratory laparotomy. Treatment success relies on a high index of suspicion and may include open operative intervention.

\section{Background}

Urovascular fistulas are well-documented in the urologic literature, although only in small case series. These usually occur between the ureter and iliac artery in patients with predisposing risk factors, such as prior pelvic surgery, radiation, chronic ureteral stents and other vascular pathologies. ${ }^{1}$ Treatment options vary widely with respect to open versus endovascular repair. While ureterovascular fistulae are welldescribed, to our knowledge there has been only 1 account of a fistula between a neobladder and artery, and endovascular management was used in this setting. ${ }^{2}$ We are reporting the second case of a neobladder-vascular fistula that was alternatively managed with an open surgical approach.

\section{Case report}

A 63-year-old male with a history of muscle invasive bladder cancer was transferred to our medical centre for a suspected ruptured neobladder. Eight months prior to this encounter, he underwent a radical cystectomy, extended bilateral pelvic lymphadenectomy and a Studer ileal neobladder, a urinary diversion using $55 \mathrm{~cm}$ of ileum. ${ }^{3}$ In the month prior to transfer, he had been hospitalized twice at outside hospitals for episodes of gross hematuria. In the first occurrence, he was managed as an outpatient with antibiotics for a suspected neobladder urinary tract infection. The subsequent episode required hospital admission and Foley catheter placement. While continuous bladder irrigation, as well as manual hand irrigation of blood clot, was required initially, this subsided after a few days. The evening prior to arrival at our institution, the patient developed acute abdominal distention, pain and respiratory distress. A peritoneal tap revealed urinary ascites, and he was immediately transferred for further management. Labs on admission included a serum creatinine of $5.6 \mathrm{mg} / \mathrm{dL}$ ( $427 \mathrm{mmol} / \mathrm{L}$ ), which was presumed to be secondary to reabsorption of urine from the peritoneal cavity. Operative exploration revealed a 2-cm laceration in the afferent limb of the ileal neobladder, likely due to traumatic neobladder catheterization/instrumentation, which was repaired in 2 layers. The afferent limb consists of a $10-$ to $15-\mathrm{cm}$ ileal chimney used for ureteral anastomosis and serves an antireflux role. Endoscopy of the neobladder was performed at that time as well; however, no sources of previous hematuria episodes were identified. During routine postoperative care, the patient's urine remained clear. On postoperative day 7 , nearing hospital discharge, he experienced an acute loss of consciousness and hemorrhagic shock preceded by massive and rapid gross hematuria. The Foley catheter that was in place since surgery was clamped to tamponade active bleeding. This allowed time for transfer to the intensive care unit, central line placement, and volume resuscitation. The patient received 4 units of packed red blood cells through a rapid infuser and follow-up labs revealed a hemoglobin and lactate of $7.2 \mathrm{~g} / \mathrm{dL}$ and $5.1 \mathrm{mmol} / \mathrm{L}$, respectively. Once hemodynamically stable, he was taken emergently to the operating room for re-exploration. No diagnostic imaging was obtained since the clinical picture strongly suggested a large vascular fistula. 
Initially, intraoperative cystoscopy was attempted to localize the bleeding source. However, this was quickly abandoned due to inadequate visualization from a large clot burden. Subsequent laparotomy revealed a tense and distended neobladder, but no evidence of bleeding into the peritoneal cavity. The afferent limb of the neobladder was opened at the same site that was repaired 1 week previously, and no acute source of bleeding was identified immediately in the neobladder. After clot evacuation, pulsatile blood flow was seen emanating from the right side wall of the neobladder, immediately adjacent to the right external iliac artery. The relative location was identified on a CT scan obtained several days prior to this incident (Fig. 1). Proximal and distal vascular control was obtained on the external iliac artery and the fistula tract was dissected. A ruptured atheromatous plaque was identified in contiguity with a hole in the neobladder. The diseased arterial segment was resected and the artery re-anastomosed primarily without graft. The neobladder was closed primarily in 2 layers, and a pedicled flap of omentum was positioning between the right external iliac artery and the neobladder. Intra-operatively, the patient received $3.8 \mathrm{~L}$ of packed red blood cells in addition to $1.2 \mathrm{~L}$ of other blood products, following our institutional massive transfusion protocol. Despite this, the postoperative labs revealed a hemoglobin of only $8.7 \mathrm{~g} / \mathrm{dL}$, emphasizing the degree of acute blood loss that had occurred. The patient recovered well and was discharged 9 days later without any lower extremity abnormalities or compartment syndrome. At the 10-month follow-up, he reported complete continence during the day with mild nighttime leakage, and there have been no untoward sequelae from this encounter.

\section{Discussion}

A fistula, by definition is an abnormal connection between 2 or more epithelial-lined organs or vessels. Fistulas between the vascular and urinary systems are not common, but have been described in several case series. The exact pathology is not well-understood; however, inflammation, fibrosis, ischemia and necrosis are the final common pathway for a multitude predisposing conditions, such as pelvic surgery, radiation therapy, vascular surgery and chronic ureteral stents. ${ }^{4}$

Ureterovascular fistulas represent most of the fistulas reported between the urinary and vascular systems. Still, the most recent review in 2008 reports only 139 cases dating back to $1939 .{ }^{5}$ Because they are so uncommon, patients presenting with recurrent bouts of gross hematuria often go undiagnosed for several weeks. This likely contributes to mortality rates as high as $58 \%$ if untreated. ${ }^{5}$ Less commonly reported are cases of fistulas between urinary conduits and arteries. ${ }^{6-10}$ In a review of 80 cases of ureterovascular fistulas, Bergqvist and colleagues reported that $36 \%$ occurred in the

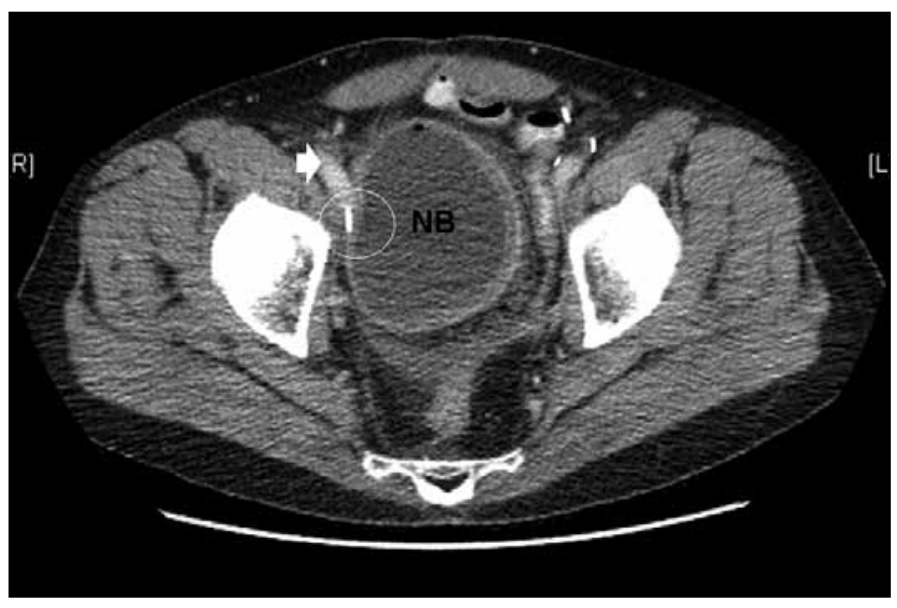

Fig. 1. Axial computed tomography scan demonstrates the location of the fistula tract (white circle) between the right external iliac artery (white arrow) and the neobladder.

setting of ileal conduits, suggesting even when a urinary diversion is present, a fistula to the ureter is more likely. ${ }^{11}$

Certainly, the least reported type of fistula is one between a neobladder and artery. In 2006, Gallucci and colleagues described a case of a 62-year-old male who, following an episode of hematuria and clot urinary retention, was diagnosed with an arterio-neobladder fistula by angiography. ${ }^{2}$ This was subsequently managed with an endovascular graft under local anaesthesia, and no blood transfusions were required. The long-term outcomes from this case were not reported.

In patients that are hemodynamically stable, endovascular approaches have been successful in several cases of ureterovascular fistulas. ${ }^{12,13}$ However, a very relevant difference is highlighted in the present case, where the bowel is in direct continuity with the fistula tract. In this situation, placement of a prosthetic graft should be avoided due to a high graft infection risk, as bowel diversions are chronically colonized. In the Mayo Clinic series of ureterovascular fistulae in 7 patients, Krambeck and colleagues recommended that endovascular stenting be used only as a temporizing measure or poor surgical candidate. ${ }^{4}$ We would agree with this recommendation, and would argue that open surgical repair is the preferred approach in the case of a neobladder-arterial fistula. Cystoscopy may be attempted first to localize the fistula to a particular ureter, but this may prolong definitive treatment and should be avoided in unstable patients. The underlying conditions that lead to fistula formation are often not modifiable, thus consideration should be given to placing a tissue flap over the arterial repair to serve as an interposing layer. This may prevent recurrence of an arterial-conduit fistula as recently reported by Hori and Thiruchelvam. ${ }^{8}$ Additional surgical treatment options include vessel ligation and subsequent extra-anatomic bypass. This method circumvents many of the predicating risk factors altogether, and is optimal for 
patients who will require continued chronic ureteral stents to maintain renal function.

As there are few occasions when the urologist is exposed to such a high acuity, stress-provoking and uncontrolled hemorrhage, the importance of having a broad knowledge of possible etiologies cannot be overstated. The presentation of our patient is very similar and even characteristic of this disease process. Gross hematuria is the sine qua non manifestation; however, this can be mild and intermittent (i.e., herald bleeding), often indicating a more common diagnosis. Batter and colleagues reported intermittent gross hematuria for up to 3 weeks prior to massive hematuria in patients ultimately diagnosed with urovascular fistulas. ${ }^{1}$ In the setting of a urinary diversion, gross hematuria generally requires some evaluation. Etiologies that are more common than fistulae include cancer recurrence (upper tracts or urethra), stones, and infection. However, these processes rarely cause the massive bleeding that can be encountered with vascular fistulas.

Unfortunately, imaging modalities have not been a reliable strategy for the diagnosis of these rare events, which is likely associated with the intermittent nature of these processes. At the initiation of the tract formation, natural clotting mechanisms may prevent frank exsanguination for a period of time. Therefore, unless active extravasation is occurring, you will not be able to identify an abnormality radiographically. Provoking this temporary clot by manipulating a ureteral stent simultaneous to angiography has been reported, but should be approached with extreme caution. ${ }^{14}$ Angiography (non-provoked) was helpful in $69 \%$ of cases, while computed tomography was helpful in $42 \%{ }^{5}$ Cystoscopy and retrograde studies were diagnostic in $60 \%$ of reported ureterovascular fistula, ${ }^{1}$ and while these were attempted in the present case, they were unsuccessful due to the large clot burden. Once the fistula tract opens definitively, rapid clinical deterioration is inevitable and imaging should not delay treatment.

\section{Conclusion}

Cystectomy patients are at risk for urovascular fistulas. These usually occur between an artery and ureter, but can also involve a direct connection to an orthotopic urinary diversion, as we have reported. As such, episodes of gross hematuria need to be evaluated appropriately, at least to exclude more common causes. This population, which are largely managed with bowel diversions, provides a unique chal- lenge to endovascular approaches, and should be treated with an open repair in most case. Presentation is often characterized by days to weeks of intermittent gross hematuria that culminate in life-threatening hemorrhage. Management may be required in the absence of radiographic diagnosis, and a high index of suspicion is paramount in this setting. Treatment should be targeted toward patient stabilization, definitive treatment of the lesion, and prevention of recurrence by incorporating omental flaps when indicated. This requires a multidisciplinary approach, including visits with a urologist, vascular surgeon and interventionist, for successful management and long-term success.

Competing interests: None declared.

This paper has been peer-reviewed.

\section{References}

1. Batter SJ, McGovern FJ, Cambria RP. Ureteroarterial fistula: case report and review of the literature. Urology 1996;48:481-9. http://dx.doi.org/10.1016/S0090-4295(96)00202-6

2. Gallucci $M$, Piccirillo $G$, Guaglianone $S$, et al. An unusual complication after a cystectomy: a case of iliac artery-neobladder fistula. Int I Urol 2006;13:1005-6.

3. Studer UE, Varol C, Danuser H. Orthotopic ileal neobladder. BJU Int 2004;93:183-93.

4. Krambeck AE, DiMarco DS, Gettman MT, et al. Ureteroiliac artery fistula: diagnosis and treatment algorithm. Urology 2005;66:990-4. http://dx.doi.org/10.1016/i.urology.2005.05.036

5. van den Bergh RC, Moll FL, de Vries JP, et al. Arterioureteral fistulas: unusual suspects-systematic review of 139 cases. Urology 2009;74:251-5. http://dx.doi.org/10.1016/i.urology.2008.12.011

6. Beaugie JM. Fistula between external iliac artery and ileal conduit. Br J Urol 1971;43:450-2. http:// dx.doi.org/10.1111/i.1464-410X.1971.tb12067.x

7. Hindmarsh JR. Common iliac-ileal conduit fistula. Br J Urol 1977;49:508. http://dx.doi.org/10.1111/ j.1464-410X.1977.tb04192.x

8. Hori $\mathrm{S}$, Thiruchelvam N. Life-threatening hematuria due to recurrent arterial-conduit fistula. Urology 2012;79:23-7. http://dx.doi.org/10.1016/j.urology.2011.08.027

9. Ishibashi H, Ohta T, Sugimoto I, et al. Successful treatment of an aorto-ileal-conduit fistula with an endovascular stent graft: report of a case. Surg Today 2007;37:305-7. http://dx.doi.org/10.1007/ s00595-006-3402-7

10. Sasaki T, Onishi T, Hoshina A. Fistula between the external liac artery and the body of an ileal conduit. Int J Urol 2011;18:260-1.

11. Bergqvist D, Parsson H, Sherif A. Arterio-ureteral fistula-a systematic review. Eur J Vasc Endovasc Surg 2001;22:191-6.

12. Gibbons $M, O^{\prime}$ Donnell $S$, Lukens $M$, et al. Treatment of a ureteroiliac artery fistula with an intraluminal endovascular graft. J Urol 1998;159:2083-4. http://dx.doi.org/10.1016/S0022-5347(01)63260-9

13. Han KR, Pantuck AJ, Siegel RL, et al. Endovascular stent graft for management of ureteroarterial fistula after orthotopic bladder substitution. Tech Urol 1999;5:169-73.

14. Keller FS, Barton RE, Routh WD, et al. Gross hematuria in two patients with ureteral-ileal conduits and double-J stents. J Vasc Interv Radiol 1990;1:69-77; discussion 69.

Correspondence: Dr. Brant Inman, Duke University Medical Center, Room 1570, White Zone, 200 Trent Drive, Durham, NC 27710; brant.inman@duke.edu 